The Astrophysical Journal, 525:L49-L52, 1999 November 1

(C) 1999. The American Astronomical Society. All rights reserved. Printed in U.S.A.

\title{
IMAGING THE HARO 6-10 INFRARED COMPANION
}

\author{
Chris D. Koresko, Geoffrey A. Blake, and Michael E. Brown \\ Division of Geological and Planetary Sciences, Caltech, Pasadena, CA 91125 \\ Anneila I. SARgent \\ Department of Astronomy, Caltech, Pasadena, CA 91125 \\ AND \\ DAVID W. KoERneR \\ Department of Physics and Astronomy, University of Pennsylvania, Philadelphia, PA 19104-6396 \\ Received 1999 August 3; accepted 1999 September 3; published 1999 October 4
}

\begin{abstract}
We present an infrared imaging study of the low-mass pre-main-sequence binary system Haro 6-10. This system is one of a handful in which the optically visible primary has the characteristics of a normal $\mathrm{T}$ Tauri star, while the secondary is a so-called "infrared companion" (IRC), a strongly extincted object that emits most of its luminosity in the infrared. A speckle holographic technique was used to produce nearly diffraction-limited images on three nights over a 1 yr period starting in late 1997. The images show that the IRC is obscured and surrounded by a compact, irregular, and variable nebula. This structure is in striking contrast to the well-ordered edge-on disk associated with HK Tauri B, the extincted companion to another T Tauri star of similar age. A new, resolved intensity peak was found $00^{\prime \prime} 4$ southwest of the IRC. We suggest that it may represent light scattered by a clump of dusty material illuminated by starlight escaping along an outflow-carved cavity in the IRC envelope. The primary star became fainter and the companion became more extended during the observing period.
\end{abstract}

Subject headings: binaries: visual — circumstellar matter — methods: miscellaneous stars: individual (Haro 6-10) — stars: pre-main-sequence

\section{INTRODUCTION}

Among the low-mass pre-main-sequence binary systems in nearby active star-forming regions, there exist a handful in which one of the two stars is reminiscent of a protostar, radiating primarily at infrared wavelengths and faint or undetected in visible light. These objects are referred to as "infrared companions" (IRCs), despite being in most cases more luminous than their primaries. Their bolometric temperatures, which measure the "center of mass" of the spectral energy distribution and are correlated with evolutionary status for young stars (Myers \& Ladd 1993), tend to lie in a transition region between true embedded sources on the one hand and classical or weak-lined T Tauri stars on the other. A variety of models have been proposed for the IRCs, but they are generally taken to be dust-shrouded stars that are either less evolved than their primaries and have yet to dissipate their natal envelopes (e.g., Dyck, Simon, \& Zuckerman 1982) or are experiencing episodes of enhanced accretion, perhaps due to interactions with the primary or with a circumbinary disk (e.g., Koresko, Herbst, \& Leinert 1997, hereafter KHL). The prototype IRC is the companion to T Tauri itself.

Early studies of Haro 6-10 by Elias (1978) revealed a nonstellar photographic appearance, a spectrum displaying prominent forbidden line emission, and photometric variations of a factor $\sim 3$ at $2.2 \mu \mathrm{m}$. The infrared companion was found 1.2 north of the visible star by Leinert \& Haas (1989, hereafter LH), who used slit-scanning speckle interferometry to measure the ratio of the brightnesses of the two stars at wavelengths between 1.65 and $4.8 \mu \mathrm{m}$. As in the T Tauri system, the IRC was fainter than the visible star at wavelengths shorter than $\sim 4 \mu \mathrm{m}$, but brighter in the mid-infrared. The Haro 6-10 IRC is the reddest object studied by KHL, with a bolometric temperature of only $210 \mathrm{~K}$, compared to $490 \mathrm{~K}$ for the T Tauri IRC.

In addition to the IRC, LH found evidence of extended emis- sion, in the form of visibility amplitude curves that fell below unity at high frequencies, for scans taken perpendicular to the axis of the binary. By contrast, scans taken along the binary axis showed only the oscillations typical of a pair of pointlike stars. LH argued that this extended emission was probably associated with one of the two stars. With the advent of twodimensional infrared detectors on large telescopes, together with refined speckle imaging techniques, it has become practical to directly image the components of young binaries to search for faint tertiary components and diffuse circumstellar material. This Letter presents the results of a speckle holographic imaging study of the Haro 6-10 IRC.

\section{OBSERVATIONS}

The new high-resolution images were taken in the $K$ $(2.2 \mu \mathrm{m})$ photometric band at the $10 \mathrm{~m}$ Keck 1 telescope on three nights in 1997 November, 1998 March, and 1998 November (see Table 1) using the Near-Infrared Camera (NIRC; Matthews \& Soifer 1994). The NIRC image converter (Matthews et al. 1996) produced a magnified pixel spacing of 0.02 , approximately Nyquist-sampling the diffraction limit at $2 \mu \mathrm{m}$. The observations consisted of thousands of exposures of the visual binary, with integration times of $\sim 0.15 \mathrm{~s}$ for each frame. This short exposure time partially "froze" the atmospheric seeing, so that the point-spread function (PSF) consisted of distinguishable speckles. The 1".2 separation of the binary was large enough that the PSFs did not significantly overlap in frames with good seeing.

Individual frames were calibrated in the standard way by subtracting mean sky frames, dividing by flat-field images, and "fixing" bad pixels. A model was computed for the "bleed" signal which extended along and perpendicular to the readout direction, and this was subtracted from the calibrated frame. For each frame, a measurement of the instantaneous PSF was made by masking all pixels on the side of the frame toward 
TABLE 1

HOLOGRAPHIC OBSERVATIONS

\begin{tabular}{cccccc}
\hline \hline $\begin{array}{l}\text { Date } \\
(\mathrm{UT})\end{array}$ & $\begin{array}{c}\lambda \\
(\mu \mathrm{m})\end{array}$ & $\tau_{\text {int }}$ & Frames & $\begin{array}{c}\text { Seeing FWHM } \\
(\operatorname{arcsec})\end{array}$ & Brightness Ratio \\
\hline 1997 Nov 22 $\ldots \ldots$ & 2.2 & 0.15 & 931 & 0.32 & $0.153 \pm 0.001$ \\
1998 Mar 8 $\ldots \ldots \ldots$ & 2.2 & 0.15 & 1276 & 0.36 & $0.295 \pm 0.001$ \\
1998 Nov 3 $\ldots \ldots \ldots$ & 2.2 & 0.14 & 2230 & 0.40 & $0.388 \pm 0.002$ \\
\hline
\end{tabular}

the IRC, leaving only the primary. Frames in which the instantaneous seeing was too poor for this procedure to cleanly separate the stars were rejected. For the rest, the Fourier power spectrum of the PSF frames and the cross spectrum (i.e., the Fourier transform of the cross-correlation) of the masked frame with the unmasked frame were computed. If the primary star is unresolved, then in principle the ratio of the cross spectrum to the PSF frame's power spectrum is the Fourier transform of the diffraction-limited image.

Raw frames produced by NIRC suffer from semicoherent electronic pattern noise, which is typically concentrated in small regions of the Fourier domain. If not corrected, this noise limits the sensitivity of the holography technique. To identify the contaminated frequencies, a noise frame was constructed by replacing the pixels containing the stars with copies of an empty region near a corner of the field. The power spectrum of the noise frame was computed, and frequencies containing more power in the noise frame than the average over the series of unmasked frames at similar frequencies were marked as bad.

The PSF power and the cross spectrum at uncontaminated frequencies were accumulated over the whole series of frames. A final image was reconstructed from them, with the use of an apodizing function to suppress high-frequency noise. The image was then rotated to standard orientation. The apodizing function chosen was the product of a Gaussian and a Hanning function. It produced a final image resolution of 81 mas (FWHM).

The resulting images for each of the three nights are presented in Figure 1. The image from each epoch is scaled to the maximum pixel level in the IRC. These images display a dynamic range, measured as the brightness of the peak of the primary star in units of noise, of $\gtrsim 2000$. This is much larger than is typical for a speckle image; the improvement is due to the holography technique's use of instantaneous PSF measurements instead of relying on a statistical consistency of the atmosphere between separate target and reference-star frames as in normal speckle interferometry. The technique does produce a residual artifact in the form of an apparent ridge of emission that runs approximately midway between the two stars and parallel to the direction of the mask used to make the PSF frames. This is probably due to a small amount of flux from the primary that spills over into the masked region. In addition, in the final epoch there is a narrow strip that extends from the IRC in a direction approximately north and exactly parallel to the detector readout.

A direct $K$-band image of the region was taken without the image converter, so that the raw field was $38^{\prime \prime}(\sim 5000 \mathrm{AU})$. Frames were taken with the binary in two well-separated locations, and the reduction was done by computing the difference between these frames and pixel-fixing. The resulting image, presented in Figure 2, has much lower resolution but higher flux sensitivity than the holographic image. It shows the two stars surrounded by an arc of nebulosity that curves gently from the primary star.
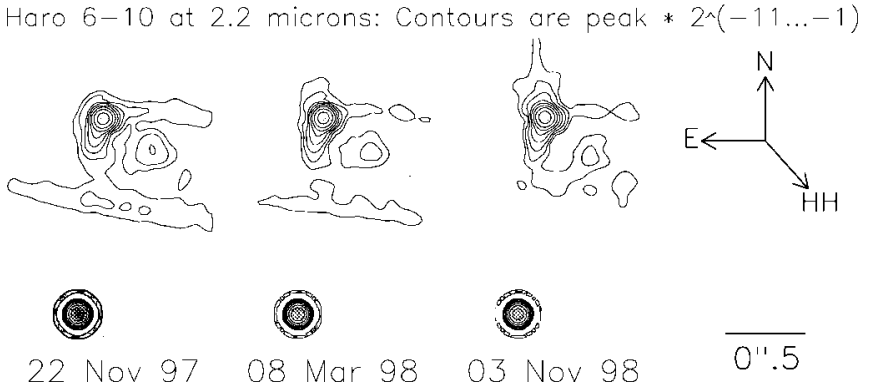

FIG. 1.-Holographic images of the Haro 6-10 system at $2.2 \mu \mathrm{m}$ taken on three nights over a $1 \mathrm{yr}$ period. They are scaled so that the peak flux in the IRC is the same for the three epochs. The stars are separated by 1.2 , and the resolution in the images is 81 mas (FWHM). The images are in standard orientation with north up, east left. The IRC's peak is resolved, and it is surrounded by a complex distribution of circumstellar matter. A bright "tail" extends to the south and, in the final epoch, a fainter one extends to the north. The nebulous peak IRC-SW lies 0".4 to the southwest of the IRC, along the direction of the giant Herbig-Haro flow (labeled "HH" in the compass rose). The primary is unresolved by assumption, and the rings around its image are intrinsic to the PSF produced by the postprocessing. The ridgelike structure between the stars and the narrow stripe extending north from the IRC in the final epoch are artifacts.

\section{RESULTS}

The Haro 6-10 infrared companion is clearly resolved in the image taken on each of the three nights. Its peak is marginally resolved in all three images, with a deconvolved FWHM of $\sim 35$ mas, or $5 \mathrm{AU}$, along cuts in the east-west direction. The peak is surrounded by a complex and irregular structure, the brightest part of which consists of a nebulous "tail" that extends some 300 mas to the south. A fainter peak, which we designate

\section{Haro 6-10 direct image ot 2.2 microns}

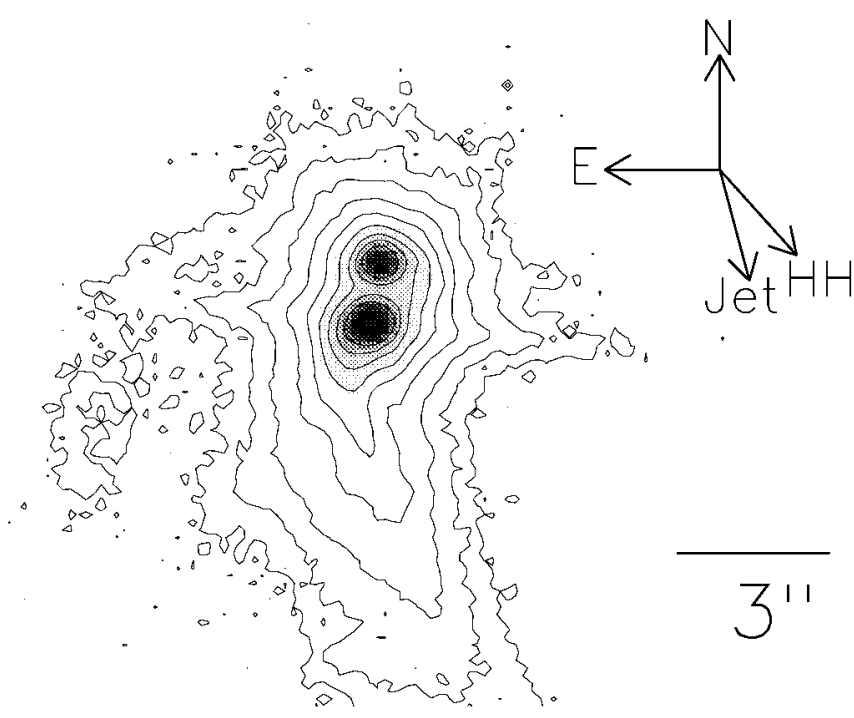

FIG. 2.-Direct image of Haro 6-10 and the IRC made by stacking four $1.0 \mathrm{~s} K$-band frames taken without the image converter. It shows the two stars and the small jet previously detected by Movessian \& Makagian (1999), which is seen to curve gently away from the primary star. Note that the faint spikes in this image are due mainly to diffraction from the hexagonal secondary spider and the edges of the segments that make up the primary mirror. The image is in standard orientation, and the contour levels are peak times $2^{-1}$ to $2^{-9}$. 
Haro 6-10 IRC-SW, appears 400 mas to the southwest of the IRC. Finally, a narrow "arm" extends 500 mas westward from a point just north of the peak.

The IRC underwent a significant morphological change during the observations, especially between the second and third epochs. Between the first and third epochs, a new "tail" appears to the north of the peak, reaching an extent of $\sim 200$ mas. It is fainter but wider than the southern tail. At the same time, IRCSW dimmed by a factor of $\sim 2$ compared to the IRC's peak.

The $2.2 \mu \mathrm{m}$ brightness of the IRC, measured as a fraction of the brightness of the primary, more than doubled from 0.15 to 0.39 (see Table 1). For comparison, $\mathrm{LH}$ found it to be 0.13 in 1988 September and KHL found 0.04 in 1994 October.

In order to compare the holographic images with the onedimensional speckle measurements by LH, we projected the holographic images along a position angle perpendicular to $355^{\circ}$, computed the one-dimensional Fourier amplitudes, and divided them by the amplitude of a projected point-source image made with the same apodization function. The first and third epochs show excellent agreement with the $K$-band amplitude curve of LH, while the middle epoch shows a small $(\sim 5 \%)$ depression in the zero-frequency level.

\section{DISCUSSION}

It would be natural to suppose that the circumstellar material surrounding the IRC may be in the form of an optically thick disk of gas and dust. Its "peak with tails" morphology in the final epoch qualitatively resembles simulations of a nearly edge-on disk (e.g., Wood et al. 1998), with the southern and northern tails tracing light scattering in the upper regions of the sunward-facing side of the disk, and the star located somewhere slightly to the west of the emission peak. Such a disk is seen in HK Tauri, in which the secondary star is completely obscured and the disk is traced at visible and near-infrared wavelengths via the starlight it scatters (Stapelfeldt et al. 1998; Koresko 1998). In the case of Haro 6-10, the disk axis would be nearly perpendicular to the line joining the two stars, as one would expect if the disk lies in the orbital plane of the binary and in contrast to the probable geometry of the HK Tauri system.

The large extinction that would be produced by an edge-on disk is consistent with previous infrared spectroscopic and spectrophotometric results. A deep mid-infrared silicate absorption feature was seen toward the IRC by van Cleve et al. (1994), while no such absorption was seen toward the primary. The low-resolution $K$-band spectrum measured by Herbst, Koresko, \& Leinert (1995) is a featureless continuum except for a molecular hydrogen $v=1-0 S(1)$ emission line at $2.12 \mu \mathrm{m}$. This suggests that the $2 \mu \mathrm{m}$ light originates primarily in dust, which may be heated either by stellar photons or by gravitational energy released via disk accretion. The lack of a detectable $v=2-1 S(1)$ line was taken to indicate that the hydrogen is excited in a shock, presumably associated with either accretion or outflow, rather than pumped by ultraviolet photons.

However, the simple edge-on disk picture alone fails to account for several important features of the system. In particular, it is not obviously consistent with the existence of Haro 6-10 IRC-SW and the west-facing arm or with the lack of a northern tail in the first epochs. If most of the circumstellar mass does reside in a disk, then it appears that the disk may have suffered strong perturbations that have disrupted its outer regions.

The timescale over which the IRC's morphology changes is too short to correspond to orbital or freefall motions in the material in the outer regions of the nebula, where the orbital period is on the order of centuries. This suggests that the changes are the result of changing illumination of the distant material due, e.g., to shadowing of the central star by material orbiting within $\sim 1$ AU of the star or by starspots (e.g., Wood \& Whitney 1998).

Additional clues to the structure of the IRC are provided by the outflows associated with the system. A giant Herbig-Haro flow which extends about $1.6 \mathrm{pc}\left(39^{\prime}\right)$ along a position angle close to $222^{\circ}$ was found by Devine et al. (1999), who suggest that the flow originates from the IRC. This interpretation may be problematic in the context of the disk model, since the outflow's position angle is $\sim 45^{\circ}$ from the sky-projected axis of the putative disk. Movessian \& Makagian (1999) report the discovery of a jet that curves away from the binary at a position angle of $195^{\circ}$, although they could not identify its source with either of the stars. This is presumably structure seen in our direct image, in which it appears to be associated with the primary.

If the IRC is the source of the giant $\mathrm{HH}$ flow, then Haro 6-10 IRC-SW lies suggestively along the flow axis. But this object shows no sign of the $\sim 300$ mas outward motion that would be expected over the $\sim 1$ yr span of the observations if it moved at the $\sim 200 \mathrm{~km} \mathrm{~s}^{-1}$ typical of the pattern speed in such a flow (e.g., Eisloffel \& Mundt 1992). By contrast, the free-fall velocity would produce an undectable motion. We conclude that IRC-SW is more likely to be orbiting the IRC or associated with envelope material than to be part of the outflow.

If IRC-SW were a stellar companion to the IRC, it could easily perturb the IRC's disk, and any disk of its own would be similarly perturbed, perhaps accounting for its photometric variability. A triple system would likely be unstable to significant orbital evolution or even ejection on timescales shorter than the age of Haro 6-10 (Pendleton \& Black 1983). On the other hand, IRC-SW's surface brightness is roughly consistent with a model in which an isotropically scattering dusty surface is illuminated by unextincted light from the central star of the IRC, which is taken to have $T \sim 5400 \mathrm{~K}$ and $L \sim 6 L_{\odot}$ (KHL). The low extinction seen by IRC-SW could result from its position along the outflow axis of the giant $\mathrm{HH}$ flow, which would clear a path through the IRC envelope, and the scattering surface would be either an irregularity in the surface of the outflow cavity or a knot of material within it. Instabilities in the outflow (Devine et al. 1999) might account for the apparent variability of the object.

\section{CONCLUSIONS}

The complex structure seen in the Haro 6-10 IRC is in stark contrast to the simple, beautifully regular shape of the nearly edge-on disk that surrounds HK Tauri B. While it would be premature to draw strong conclusions from this about the IRC class as a whole, it does suggest that at least in this object the characteristic low infrared color temperature may be the product of reprocessing and scattering in a disk that has been strongly perturbed, at least in its outer regions. The origin of this perturbation is not clear, but possibilities include interactions with the giant Herbig-Haro flow, with residual infalling cloud material, or with a possible star embedded in IRC-SW.

It is a pleasure to thank A. Bouchez for his assistance with the observations, F. Roddier for a useful suggestion regarding the data reduction, and the anonymous referee for suggestions 
that improved the interpretation of the results. Data presented herein were obtained at the W. M. Keck Observatory, which is operated as a scientific partnership among the California Institute of Technology, the University of California, and the
National Aeronautics and Space Administration. The Observatory was made possible by the generous financial support of the W. M. Keck Foundation. This research was supported by the National Aeronautics and Space Administration.

\section{REFERENCES}

Devine, D., Reipurth, B., Bally, J., \& Balonek, T. J. 1999, AJ, 117, 2931

Dyck, H. M., Simon, T., \& Zuckerman, B. 1982, ApJ, 255, L103

Eisloffel, J., \& Mundt, R. 1992, A\&A, 263, 292

Elias, J. H. 1978, ApJ, 224, 857

Herbst, T. M., Koresko, C. D., \& Leinert, C. 1995, ApJ, 444, L93

Koresko, C. D. 1998, ApJ, 507, L145

Koresko, C. D., Herbst, T. M., \& Leinert, C. 1997, ApJ, 480, 741 (KHL)

Leinert, Ch., \& Haas, M. 1989, ApJ, 342, L39 (LH)

Matthews, K., Ghez, A. M., Weinberger, A. J., \& Neugebauer, G. 1996, PASP, 108,615
Matthews, K., \& Soifer, B. T. 1994, Infrared Astronomy with Arrays: The Next Generation, ed. I. McLean (Dordrecht: Kluwer), 239

Movessian, T. A., \& Makagian, T. Y. 1999, A\&A, 347, 266

Myers, P. C., \& Ladd, E. F. 1993, ApJ, 413, L47

Pendleton, Y. J., \& Black, D. C. 1983, AJ, 88, 1415

Stapelfeldt, K. R., Krist, J. E., Menard, F., Bouvier, J., Padgett, D. L., \& Burrows, C. J. 1998, ApJ, 502, L65

van Cleve, J. E., Hayward, T. L., Miles, J. W., Gull, G. E., Schoenwald, J., \& Houck, J. R. 1994, Ap\&SS, 212, 231

Wood, K., Kenyon, S. J.,Whitney, B., \& Turnbull, M. 1998, ApJ, 497, 404 Wood, K., \& Whitney, B. 1998, ApJ, 506, L43 